\title{
Le divorce dans le théâtre révolutionnaire: du motif dramatique nouveau au discours théâtral sur la loi et ses usages
}

Philippe Corno

\section{(2) OpenEdition}

Journals

Édition électronique

URL : http://journals.openedition.org/studifrancesi/3283

DOI : $10.4000 /$ studifrancesi.3283

ISSN : 2421-5856

Éditeur

Rosenberg \& Sellier

Édition imprimée

Date de publication : 1 avril 2013

Pagination : 63-73

ISSN : 0039-2944

Référence électronique

Philippe Corno, «Le divorce dans le théâtre révolutionnaire: du motif dramatique nouveau au discours théâtral sur la loi et ses usages », Studi Francesi [En ligne], 169 (LVII | I) | 2013, mis en ligne le 30

novembre 2015, consulté le 18 septembre 2020. URL : http://journals.openedition.org/studifrancesi/ 3283 ; DOI : https://doi.org/10.4000/studifrancesi.3283

\section{(c) $($ ) $(9)$}

Studi Francesi è distribuita con Licenza Creative Commons Attribuzione - Non commerciale - Non opere derivate 4.0 Internazionale. 


\section{Le divorce dans le théâtre révolutionnaire: du motif dramatique nouveau au discours théâtral sur la loi et ses usages}

\section{Résumé}

La rupture du lien conjugal est légalisée pour la première fois en France le 20 septembre 1792. À travers l'étude de quelques pièces significatives, cet article évoque le traitement que le théâtre révolutionnaire a réservé à cette question du divorce (les débats, la loi puis les pratiques). Il montre combien ce sujet a pu renouveler dans une certaine mesure le fonds thématique dont le théâtre révolutionnaire a hérité du XVIII ${ }^{\mathrm{e}}$ siècle; mais surtout il démontre que la question du divorce au théâtre interrogeait alors la place du mariage dans la société française et engageait plus largement celle de ce théâtre sur la scène de la Révolution.

Le théâtre peut être considéré comme la représentation qu'une société se donne d'elle-même à elle-même; cette idée, fondamentale pour appréhender la place de cet art dans la cité, n'implique nullement qu'il soit une sorte de miroir fidèle que les dramaturges tendraient à la société, pas plus qu'il ne serait, plus anachroniquement, la photographie d'un monde extérieur qui viendrait prendre vie, le temps de la représentation, sur le bois de ses planches. Ce que nous montre la scène ne saurait être appréhendé comme l'extrait - comme l'échantillon, pourrait-on dire - d'une vie quotidienne toujours difficile à saisir pour l'historien. Pour autant, il constitue un formidable révélateur des représentations individuelles et collectives, pourvu qu'on se donne du temps pour penser ce qu'un dispositif théâtral, une esthétique dramatique, un type de décors, de costumes ou encore une manière de jouer a à nous dire sur les structures idéologiques, politiques, juridiques, sociologiques, sexuelles, etc. d'une société donnée. En ce sens, le théâtre d'une nation, comme le théâtre d'une époque, révèle toujours un peu de son essence, un peu de ce qui la singularise et la définit en propre.

Le théâtre de la Révolution française ne fait assurément pas exception à ce principe; au contraire, plus que d'autres peut-être, il s'est fait l'écho des rumeurs du monde qui l'a vu naître. À cela, il est plusieurs raisons que je ne ferai qu'évoquer. Héritier du drame bourgeois et de sa volonté d'être davantage en prise avec son époque, le théâtre révolutionnaire va fréquemment chercher ses sujets dans l'actualité la plus immédiate. Et il faut bien reconnaître que les événements révolutionnaires, bien souvent déjà dramatiques en eux-mêmes, ne se montrent pas avares en sujets nouveaux, variés et puissants. Il n'est pas jusqu'au foisonnement, jusqu'à la rapidité du renouvellement des créations qui, en soutenant l'innovation, l'émulation et même la concurrence entre les salles ${ }^{1}$, ne favorisent à leur manière cette esthétique de l'immédiat, cette écriture proche du quotidien. Enfin, la volonté des révolutionnaires eux-mêmes, qui souhaitent faire du théâtre non seulement un média de masse ouvert au plus grand nombre mais aussi une école morale et politique du peuple, va pareillement dans ce sens dans la mesure où une telle ambition suppose de préférence une

(1) La loi de libéralisation des entreprises des spectacles date du 13 janvier 1791. 
grande proximité entre le monde représenté de la fiction et le quotidien des spectateurs. Ainsi nombre des œuvres de la période révolutionnaire puisent leur sujet dans l'actualité la plus brûlante, à tel point qu'il semble difficile de trouver un événement ou une réforme révolutionnaires d'importance même moyenne qui n'ait pas fait le sujet d'une pièce: et le tutoiement, les clubs, la spéculation, les héros de la Révolution, etc. d'avoir considérablement renouvelé le fonds thématique des dramaturges.

Pour ce qui me concerne, je vais m'intéresser plus spécifiquement à la loi du divorce votée en 1792 et à la façon dont le théâtre révolutionnaire a rendu compte de ce bouleversement du droit conjugal français. Après m'être attaché à décrire les modes d'intégration de cette problématique dans les intrigues dramatiques, je me pencherai plus précisément sur la contribution de ces œuvres elles-mêmes au vaste mouvement de régénération de la matrimonialité qui traverse la période révolutionnaire. Néanmoins, avant d'évoquer la naissance de ce nouveau thème dramatique, quelques rappels d'histoire du droit paraissent nécessaires.

L'institution du mariage indissoluble dont hérite le XvIII ${ }^{e}$ siècle trouve son origine dans le quatrième concile de Latran (1215) qui, l'élevant au rang de sacrement, en fait une union bénie par le prêtre / Dieu. Cette conception d'un mariage que seule la mort peut rompre sera confirmée par la $24^{\text {ème }}$ et dernière session du concile de Trente (1563), qui en outre renforcera le contrôle sur cette institution. Si ce concile ne fut jamais reçu en France, nombre de ses principes furent directement transposés en droit français, ecclésiastique et royal. Ainsi, sans entrer dans le détail d'une histoire parfois complexe au cours de laquelle le pouvoir civil a progressivement supplanté le pouvoir religieux, il est possible de définir le mariage à la veille de la Révolution française $^{2}$ comme un sacrement et un contrat indissolubles ${ }^{3}$ qui, bien qu'unissant deux individus, se fonde plus volontiers sur les intérêts des lignées familiales que sur les sentiments des époux. Effectivement, dans le dispositif juridique qui organise l'union conjugale, l'indissolubilité contribue au même titre que d'autres principes à faire du mariage un instrument de contrôle et de gestion de la puissance patrimoniale, économique et symbolique des familles, instrument placé entre les mains du chef de famille qui figure d'une certaine manière l'autorité absolue du roi dans l'espace domestique. Ainsi, pour pouvoir convoler, les garçons de moins de 30 ans et les filles de moins de 25 ans devaient nécessairement obtenir le consentement de leurs parents; passé cet âge, ils leur devaient une sommation respectueuse; sans quoi, ils risquaient des condamnations civiles et criminelles particulièrement faites pour les dissuader de tenter l'aventure ${ }^{4}$. Mais, plus coercitif encore, l'arsenal juridique permettait aux parents d'exhéréder un enfant majeur dont ils considéraient le mariage comme une injure grave et déshonnête faite à leur famille. Ainsi armés, les pères pouvaient aisément contraindre leur descendance à des choix matrimoniaux dans l'intérêt de la lignée

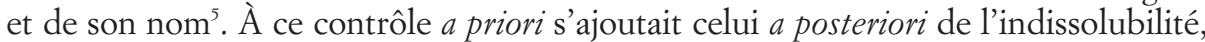
l'impossibilité de rompre leur mariage enlevant aux enfants toute possibilité de revenir sur la décision parentale en divorçant et en se remariant selon leur gré. C'est

(2) Il faudrait excepter les protestants auxquels un édit reconnaît dès 1787 un état civil, ce qui revient par voie de conséquence à établir en France un mariage civil.

(3) Il existait une possibilité d'annulation du mariage pour vice de forme ou pour défaut intrinsèque de l'union; mais ces recours étaient fort peu utilisés car ils s'avéraient longs et coûteux. De plus, dans ce cas, le mariage est annulé (il n'a jamais existé) et non rompu (il n'existe plus).
(4) Ces mariages, assimilés à des rapts de séduction, pouvaient entraîner l'annulation de l'union, l'exhérédation, la révocation des donations passées voire la peine de mort pour le présumé séducteur.

(5) D'autres pratiques et d'autres lois, comme les lettres de cachet ou comme le droit d'aînesse qui ne s'appliquait au sens strict qu'aux nobles mais qu'on retrouvait dans certains droits coutumiers, favorisaient également ces stratégies patrimoniales autour du mariage. 
peu dire alors que le mariage d'amour, s'il pouvait toujours exister - soit que des parents se montrassent à l'écoute des sentiments de leurs enfants soit aussi parfois que l'amour suivit le chemin de la raison parentale-, ne constituait pas la norme; que les futurs époux s'appréciassent était alors un bien mais assurément pas une raison ni même une condition au mariage. Les filles et les fils devaient donc apprendre à obéir plutôt qu'à suivre leur cœur; il est vrai que le droit les y aidait beaucoup... Le théâtre révolutionnaire rend d'ailleurs compte de cette situation en mettant régulièrement en scène des couples mariés contre leur gré ou, tout au moins, sans amour. Pour n'en prendre qu'un exemple, songeons à la comédie de Pigault-Lebrun, Charles et Caroline ou les Abus de l'Ancien Régime (1790)' . Dans cette pièce, au titre tout à fait explicite, Charles de Verneuil a épousé par amour, et contre l'avis paternel, Caroline, une femme sans bien; puis il s'est enfui en France pour échapper aux poursuites. Alors qu'il est revenu dans son pays avec sa femme et son fils, il manque de se faire arrêter par son père qui, conseillé par le fourbe comte de Derval, obtient une lettre de cachet contre le rebelle. Finalement, la pièce se termine heureusement: le traître est démasqué et le père finit par reconnaître le mariage en même temps que la vertu de son fils. Il n'en reste pas moins que cette pièce, qui donne clairement raison aux enfants contre les parents, témoigne distinctement des moyens de rétention législatifs dont pouvait disposer un père de famille au dix-huitième siècle pour décider des mariages de sa progéniture.

Enfin, pour brosser un tableau un tant soit peu complet des cadres juridiques du mariage et des pratiques qui en découlaient, il convient de dire un mot des rapports entre les hommes et les femmes au sein du couple. De ce point de vue, les choses paraissent assez simples, dès lors que la femme n'avait peu ou prou aucun pouvoir réel, que ce soit sur son patrimoine, ses enfants ou son couple. Comme le rappelle Dominique Godineau, «le droit coutumier français place l'épouse sous l'autorité de son mari, sans l'accord duquel elle ne peut agir»' . Le droit qui organisait les séparations $^{8}$ témoigne distinctement de cette inégalité sexuelle; elle pouvait en effet être prononcée en cas de risque de mort pour l'un des époux ou en cas d'adultère de la femme. Dans cette dernière circonstance, l'épouse était condamnée à rester enfermée au couvent à vie, dès lors que son conjoint ne la reprenait pas auprès de lui. Et si la jurisprudence s'est considérablement assouplie au cours du siècle au bénéfice du sexe féminin, la violence des maris dans les milieux populaires ainsi que l'adultère masculin' dans tous les milieux demeuraient souvent impunis. Passant ainsi des mains de leur père à celles de leur époux, les femmes restent donc toujours à la veille de la Révolution des éternels mineurs; et le mariage, malgré des appels de plus en plus pressants à la bienveillance parentale et, sinon à l'amour, du moins à la tendresse conjugale, demeure une institution fondamentalement phallocratique au service des familles.

La Révolution française va faire souffler un vent de renouveau sur ce monument juridique hérité des siècles passés. En effet, dans leur ambition de régénérer la société et de rompre avec les abus de l'Ancien Régime, les législateurs révolutionnaires ne pouvaient manquer de s'intéresser au droit conjugal. Convaincus que la famille, telle une petite patrie, devait se fonder sur les mêmes principes que ceux qui guidaient la

(6) Ch. Pigault-Lebrun, Charles et Caroline ou les Abus de l'Ancien Régime, Paris, Cailleau, 1790, pp. IV-111; comédie en cinq actes représentée pour la première fois à Paris sur le théâtre du PalaisRoyal le 28 juin 1790.

(7) D. Godineau, La Femme, in L'Homme des Lumières, M. Vovelle (éd.), Paris, Seuil, 1996, pp. 431-466.

(8) La séparation de corps et/ou de biens n'est pas un divorce; même la séparation prononcée, le lien conjugal demeure et empêche tout remariage jusqu'au décès d'un des conjoints.

(9) Sauf s'il recevait sa maîtresse au domicile conjugal. 
révolution politique alors en $\operatorname{cours}^{10}$, ils mirent en place les cadres juridiques permettant un renouveau de cette dernière. La loi sur le divorce, votée un jour avant l'abolition de la royauté ${ }^{11}$, occupe une place sinon centrale du moins primordiale dans cet édifice juridique que le Code civil détricotera substantiellement. Non seulement les législateurs inventent le droit de rompre une union conjugale - désormais considérée sous son seul versant civil - et de convoler une seconde fois, mais en plus ils accordent cette possibilité avec une très grande libéralité, sans distinction de sexe. C'est peu dire que cette loi ébranle en profondeur l'institution familiale mise en place par l'Ancien Régime, qui perd là ses fondements juridiques les plus essentiels. Dans le même esprit, on pourrait évoquer la suppression des vœux religieux ${ }^{12}$, la fixation de la majorité légale à 21 ans $^{13}$, âge au-delà duquel les enfants peuvent se marier sans l'avis de leurs parents ${ }^{14}$, l'abolition du droit de primogéniture ${ }^{15}$, l'abolition des lettres de cachet $^{16}$, l'imposition de l'égalité successorale ${ }^{17}$, etc.; mais finalement, la loi du divorce presque à elle seule enlève tout pouvoir aux pères: à quoi bon contraindre un enfant à un mariage de raison s'il est en droit de le rompre dès le lendemain? Désormais, en France, les conditions du mariage d'amour paraissent rassemblées. De même, au sein du couple, un rééquilibrage très net s'est opéré en faveur des épouses maintenant capables - au sens juridique du terme - de tenir tête à leur époux grâce au divorce. Concrètement, cette révolution de la matrimonialité s'appuie sur les conditions fixées par le législateur à la rupture des liens conjugaux. La loi sur le divorce, telle que mise en place par ses 45 articles, autorise le divorce pour trois types de raisons: le consentement mutuel, l'incompatibilité d'humeur ou de caractère, une cause déterminée $e^{18}$. Dans les faits, et sans entrer dans les détails des procédures de ces différents modes de divorce (qui ne sauraient excéder douze mois), il est important de noter que seuls les divorces pour une cause déterminée, nécessitant l'établissement de cette cause, sont judiciaires, c'est-à-dire font l'objet d'un jugement contradictoire et donc d'une décision de justice; tous les autres modes de divorce sont de droit et peuvent donc être obtenus dès lors qu'au moins un des deux époux en manifeste la volonté, et ce indépendamment du sexe du demandeur. Ainsi non seulement il est possible pour la première fois de divorcer en France, mais en outre jamais plus il ne sera aussi facile et rapide de le faire.

Pour autant, avant même d'envisager la manière dont le théâtre se saisit de ce nouveau droit conjugal, il faut bien se garder de se méprendre sur le sens d'une telle révolution juridique; la distance menace toujours le regard de l'historien de distorsions très préjudiciables à son enquête. En effet, il n'était aucunement question pour les législateurs révolutionnaires, comme la littérature contre-révolutionnaire a pu le soutenir jusqu'au siècle précédent, de détruire l'institution familiale. Bien au contraire, il s'agissait dans leur esprit de la renforcer en la refondant sur les principes de la liberté et de l'égalité; à la famille d'Ancien Régime construite comme une

(10) D’après les législateurs eux-mêmes: «L'Assemblée Nationale, considérant combien il importe de faire jouir les Français de la faculté du divorce, qui résulte de la liberté individuelle, dont un engagement indissoluble serait la perte» (préambule de la loi de 1792, in Archives parlementaires de 1797 à 1860: recueil complet des débats législatifs et politiques des Chambres françaises, M. J. MaDIVAL et M. E. LAURENT (éds.), Paris, Dupont, 1884, première série, 1787 à 1799 ).

(11) Le 20 septembre 1792.

(12) Le 13 février 1790.

(13) Loi des 16-24 août 1790.
(14) Loi des 20-25 septembre 1792

(15) Décret des 15-18 mars 1790.

(16) Le 26 juin 1789.

(17) Lois du 8 avril 1791, puis du 6 janvier 1794

(18) Ces causes sont: la démence, la folie ou la fureur d'un des époux; la condamnation de l'un d'eux à une peine afflictive ou infamante; des crimes, sévices ou injures graves à l'égard de l'un envers l'autre; le dérèglement de mœurs notoire; l'abandon d'un des époux par l'autre pendant au moins deux ans; l'absence de l'un d'eux, sans nouvelles, pendant au moins cinq ans; l'émigration. 
royauté dans laquelle chacun se trouvait soumis au pouvoir absolu du père seul dépositaire du devenir de la lignée, ils ont voulu substituer une famille rassemblant des volontés libres et supposées responsables, organisant eux-mêmes leur destin collectif dans les tribunaux et assemblées de famille. Pour tout dire, les législateurs espéraient fondamentalement que la loi sur le divorce renforcerait les liens du mariage, qu'elle rendrait - après un nécessaire effet de rattrapage - les divorces rares; ils en attendaient la régénération de la cellule familiale, sans laquelle celle de la nation ne saurait s'accomplir totalement.

Très vite, le théâtre révolutionnaire va s'emparer de la question du divorce pour en faire un matériau dramatique nouveau. Il s'agit alors d'une thématique presque inédite dans la mesure où jamais auparavant il n'avait été possible de divorcer en France et où le théâtre ne l'avait presque jamais imaginé - le quotidien étant la source où bien souvent il puise son inspiration. En effet, la quasi totalité des pièces évoquant le sujet du divorce avant la Révolution n'abordent en réalité que celle de la séparation ${ }^{19}$. Par contre, dès lors que le divorce s'inscrit dans la réalité quotidienne des Français, d'abord comme une loi désormais possible à partir de 1789, puis comme un fait juridique à partir de 1792, il se glisse sous la plume des dramaturges et sur la scène des théâtres. Plusieurs facteurs prédisposaient cet objet juridique à devenir une thématique dramatique. Retenons tout d'abord, et c'est sans doute le plus évident, son lien immédiat avec les questions de l'amour et du mariage qui sont au cœur de l'immense majorité de la production théâtrale depuis plusieurs siècles. Ainsi, faire envisager ou prononcer un divorce par ses personnages a pu aisément devenir pour les dramaturges un moyen de renouveler le fonds des intrigues dont ils ont hérité. Et cela d'autant plus avantageusement - c'est là une deuxième raison du succès de cette thématique - que le divorce a par nature quelque chose à voir avec le théâtre dans la mesure où il suppose une conflictualité hautement dramatique en elle-même. A sans doute également largement contribué à la théâtralisation du divorce la volonté pédagogique, très sensible pendant la période révolutionnaire, de mettre en place un théâtre accessible au peuple et centré sur le quotidien de ce dernier. Effectivement, de la même manière que la Révolution a souhaité placer le peuple en situation de se prendre en main, elle a voulu en faire le personnage principal de sa dramaturgie; dans ces conditions, la loi sur le divorce devant assurer la régénération du mariage et de la famille au sein de ce peuple, il n'est guère étonnant que le théâtre l'ait évoquée.

Il existe donc ce qu'on peut appeler un théâtre révolutionnaire du divorce; néanmoins les productions de ce corpus s'avèrent, selon la place et le sens que les dramaturges donnent à ce fait juridique, assez disparates. Il est cependant possible d'en esquisser une sorte de typologie capable de mieux rendre compte de cet ensemble. Tout d'abord, nous trouvons des pièces qui ne rencontrent le divorce que ponctuellement, au détour d'une réplique, sans incidence réelle sur le déroulement de l'intrigue. Ainsi en va-t-il par exemple de la pièce de Carbon de Flins, Le Réveil d'Épiménide ${ }^{20}$. qui raconte le réveil d'un homme du XVII ${ }^{\mathrm{e}}$ siècle dans le Paris révolutionnaire et dans laquelle, à la fin, l'amant de l'héroïne constate, alors qu'il est sur le point de s'unir à elle, que sa jalousie ne saurait être guérie par le mariage dès lors que le divorce est désormais permis. Dans ce cadre, la loi du divorce apparaît presque comme un élément

(19) Il faudrait excepter ici une pièce de Regnard qui invente une arlequinade dans laquelle un véritable divorce est prononcé (J.-F. REGNARD, Le Divorce, in Euvres complètes de Regnard, Paris, Lequien, 1820, tome 5, pièce jouée sur le Théâtre Italien le 16 mars 1688).
(20) C.-M.-L.-E. Carbon de Flins des Oliviers, Le Réveil d'Épiménide, Paris, Maradan, Nantes, chez Louis, Bruxelles, Le Charlier, 1790, comédie en un acte en vers, représentée sur le Théâtre de la Nation le $1^{\text {er }}$ janvier 1790. 
de décor langagier permettant aux spectateurs de reconnaître une époque, un pays ou un milieu social; dans cet usage, sa mention permet en particulier aux dramaturges de faire un lien entre les personnages d'une pièce et les spectateurs dans l'univers desquels le divorce est désormais rentré. En ce sens, il peut servir à évoquer la société héritée de la Révolution, qu'il s'agisse de celle - fût-elle fantasmée - dans laquelle vivent les spectateurs ou de celle que le dramaturge est encore réduit à imaginer parce qu'elle n'existe pas encore. On pourrait songer ici à des œuvres comme L'An 2000 de Rétif de la Bretonne ${ }^{21}$, à La Journée du Vatican ${ }^{22}$ de Giraud ou encore à Tarare ${ }^{23}$ de Beaumarchais, pièces dans lesquelles la mention de la légalité du divorce connote comme d'autres éléments le passage de la société de l'Ancien Régime à une nouvelle ère ouverte par la Révolution. Notons que ces évocations ponctuelles et anecdotiques du divorce (comme loi ou comme pratique), bien que sans incidence sur les intrigues, s'avèrent finalement assez rarement neutres et sans arrière-discours. En effet, à bien les considérer, elles fonctionnent la plupart du temps comme des marqueurs idéologiques ou moraux. Elles sont le moyen pour le dramaturge non pas de dire explicitement ce qu'il pense de la loi, mais plutôt de situer axiologiquement un personnage selon qu'il rejette ou non cette loi - sa valeur positive ou négative intrinsèque devant s'imposer, par connivence, aux yeux des spectateurs. Ainsi, dans des œuvres comme Christophe Dubois ${ }^{24}$ de Léger ou Les Mours du jour ou l'École des jeunes fermmes ${ }^{25}$ de Collin d'Harleville, il suffit qu'un divorce soit un instant envisagé ou évoqué pour que soit incidemment confirmée la déchéance morale d'un propriétaire peu recommandable et fourbe ou d'un milieu social parisien corrompu autant que corrupteur.

Dès lors qu'il suppose des motivations, un mode de réalisation et des conséquences, le divorce peut aussi être mobilisé par les auteurs non plus comme simple référence à un contexte socio-politique mais comme un des éléments constitutifs de l'intrigue, voire même comme un moyen de l'action dramatique. Les dramaturges ne se sont d'ailleurs pas privés de construire des histoires mises en place et nouées avant même le lever du rideau par la prononciation d'un divorce. Dans ce cadre, il ne s'agit alors pas tant de fouiller la dramaturgie conflictuelle du divorce en tant que telle mais bien d'en creuser les conséquences possibles, et éventuellement de les pousser jusqu'à leur terme le plus sombre (Amar du Rivier, Les Suites et les dangers du divorce $\left.{ }^{26}\right)$ ou, inversement, de les refuser en revenant sur le divorce prononcé (Desforges, Les Époux divorcés ${ }^{27}$; Kotzebue, Misantropie et repentir ${ }^{28}$ ). De ce point de

(21) N.-E. Rétif de la Bretonne, L'An 2000, édité dans Le Thesmographe ou Idées d'un bonnête bomme..., La Haye, Gosse jr. et Changuion, Paris, Maradan, 1789 , pp. 515-556, réédition Strasbourg, Heitz, «Bibliotheca romanica», s.d., 9.

(22) GIRAuD, La Journée du Vatican ou le Mariage $d u$ Pape, Turin, de l'imprimerie aristocratique, aux dépens des réfugiés français, 1790, comédie-parade en trois actes et en prose, représentée à Paris sur le Théâtre des Amis de la Patrie le 15 août 1793 [informations éditoriales fictives, pièce probablement imprimée en 1793].

(23) P.-A. Caron de Beaumarchais, Tarare, Paris, Lormel, 1787, réédition modifiée Paris, Lormel, 1790, réédité par Pierre Larthomas avec la collaboration de Jacqueline Larthomas, dans P.-A. CAron de Beaumarchais, Euvres, Paris, Gallimard, «Bibliothèque de la Pléiade», 1988, opéra en cinq actes et en vers avec un prologue, représenté pour la première fois le 8 juin 1787 à l'Académie royale de musique.
(24) F. LÉger, Christophe Dubois, Paris, Théâtre du Vaudeville, an III (1794-1795), fait historique en un acte et en prose mêlé de vaudevilles, représenté pour la première fois à Paris sur le Théâtre du Vaudeville le 21 Vendémiaire an III (12 octobre 1794).

(25) J.-F. Collin D'Harleville, Les Moeurs du jour ou l'École des jeunes femmes, Paris, Huet et Charon, an VIII (1800), comédie en cinq actes et en vers, représentée pour la première fois sur le Théâtre Français de la République le 7 thermidor an VIII (26 juillet 1800).

(26) J.-A. Amar DU Rivier, Les Suites et les dangers du divorce, édité aussi sous le titre Le Double divorce ou les Dangers de l'abus, Paris, marchands de nouveautés, an VI (1797-1798), drame en trois actes et en vers.

(27) P. J.-B. Choudard dit Desforges, Les Époux divorcés, comédie en trois actes et en vers, représentée à Paris sur le Théâtre de la Cité-Variétés le 7 fructidor an VII (24 août 1799).

(28) A. Kotzebue, Menschenhass und Reue, Ber- 
vue, ces œuvres sont davantage centrées sur la possible / l'impossible réconciliation entre les époux que sur la volonté de divorcer en tant que telle; l'état de séparation conjugale y figure une donnée préalable à l'action, sur laquelle les personnages accepteront ou non de revenir. Mais l'évocation du divorce peut aussi non pas servir la mise en place de l'intrigue mais contribuer à son développement. En ce sens, il n'appartient plus à la pré-histoire de l'œuvre mais constitue un élément de l'action à part entière, même si cette dernière ne se réduit pas à lui. Il représente alors un ressort du drame comme l'est plus traditionnellement le mariage. Ainsi, les héroïnes de la pièce de Duval, Le Tyran domestique ou l'Intérieur d'une famille ${ }^{29}$, et de celle de Pixerécourt, Avis aux femmes ou le Mari colère ${ }^{30}$, envisagent-elles ou, du moins, font-elles semblant d'envisager un divorce pour ramener leur époux à un comportement plus bienveillant à l'égard d'elles-mêmes et de leur famille. Dans une perspective similaire, l'évocation du divorce peut apparaître conjointement à d'autres enjeux (l'adhésion à la Révolution, le mariage d'un enfant, etc.) avec lesquels il forme un ensemble idéologique ou moral cohérent. Bien que non essentiel dans l'intrigue, il en constitue néanmoins l'un des piliers narratifs et dramatiques importants. C'est le cas d'une œuvre comme celle de Laya intitulée L'Ami des lois' ${ }^{31}$; en effet, si cette pièce concentre son propos autour de la vertu du révolutionnaire modéré qu'est l'aristocrate Forlis, elle n'en est pas moins introduite par l'évocation du divorce des Versac, divorce qui sera finalement refusé comme sont rejetés au dénouement de l'œuvre tous les comportements emportés et égoïstes dont la Révolution a accouché - la pièce sera pour cette raison accusée de modérantisme. Ici, le divorce ne constitue pas le propos central de l'intrigue mais néanmoins il s'y agrège de manière tout à fait logique en lui donnant davantage d'épaisseur et de portée, en soulignant le croisement des enjeux privés et publics autour de l'adhésion aux valeurs révolutionnaires. Une telle analyse pourrait valoir également pour La Mère coupable $e^{32}$ de Beaumarchais, drame dans lequel la question du divorce, bien que secondaire par rapport à l'enjeu principal de l'intrigue (l'exhérédation de Léon pour favoriser Florestine), n'en recoupe pas moins celle de l'adhésion du comte Almaviva à certaines valeurs politiques, son refus de divorcer et sa réconciliation conjugale s'y accordant distinctement avec le rejet de Begearss, auteur du projet divorciaire et incarnation de la face corrompue de la Révolution, au profit du jeune Léon, l'ardent et vertueux révolutionnaire.

Parfois enfin le divorce de tel ou tel personnage peut constituer le propos central d'une œuvre. Comme l'indiquent explicitement les titres des œuvres en question - Le Mur mitoyen ou le Divorce manque $e^{33}$, Le Divorce ${ }^{34}$, Le Double Divorce ou le Bienfait

lin, 1789, adapté par Molé sous le titre Misantropie et repentir, an VII (1798-1799), représentée pour la première fois le 26 décembre 1798.

(29) A. Duval, Le Tyran domestique ou l'Intérieur d'une famille, Paris, Vente, an XIII (1805), comédie en cinq actes et en vers, représentée pour la première fois à Paris sur le Théâtre Français le 27 pluviôse an XIII (16 février 1805).

(30) R. Ch. G. DE Pixérécourt, Avis aux femmes ou le Mari colère, Paris, Barba, an XIII (1804), comédie en un acte et en prose mêlée d'ariettes, représentée pour la première fois sur le théâtre de l'Opéra-comique national le 5 brumaire an XIII (27 octobre 1804).

(31) J.-L. LAYA, L'Ami des lois, Paris, Maradan, 1793, comédie en cinq actes et en vers, représentée par les comédiens de la Nation le 2 janvier 1793.

(32) P.-A. Caron de Beaumarchais, L'Autre Tar- tuffe ou la Mère coupable, Paris, Maradan, an II (1793), réédition Paris, Rondonneau, 1797, drame en cinq actes et en prose, représenté pour la première fois à Paris sur le Théâtre du Marais le 26 juin 1792, repris après modifications à Paris sur le Théâtre Feydeau le 16 floréal an V (5 mai 1797).

(33) P.-Y. Barré et N. BourgueIL, Le Mur mitoyen ou le Divorce manqué, Paris, Barba, an $\mathrm{X}$ (1802), comédie-vaudevilles en un acte et en prose, représentée pour la première fois à Paris sur le Théâtre du Vaudeville le 3 ventôse an IV (22 février 1796).

(34) Ch. A. Demoustier, Le Divorce, Paris, Maradan, an III (1794-1795), comédie en deux actes et en vers, représentée pour la première fois à Paris sur le Théâtre Feydeau le 10 juillet 1791; G. F. DEsFontaines, Le Divorce, Paris, Brunet, an II (17931794), comédie en un acte et en vaudevilles, repré- 
de la loi $i^{35}$, La Nécessité du divorce ${ }^{36}$, etc. - l'histoire se concentre alors généralement autour des difficultés conjugales d'un couple et de la solution que la loi du divorce pourrait y apporter définitivement. Ces pièces se construisent peu ou prou comme les habituelles comédies autour d'un mariage; les personnages s'y divisent entre ceux qui œuvrent pour la réconciliation du couple et ceux qui, bien souvent par intérêt personnel, favorisent au contraire leur dissension; et l'intrigue de s'achever par une ré-union des époux, avec ou sans chansons. Ces créations apparaissent souvent comme les plus intéressantes pour qui étudie les représentations de la loi du divorce sur la scène dans la mesure où, abordant plus frontalement et plus longuement cette problématique, elles prennent le temps d'exposer autour du conflit conjugal ce qui peut motiver un divorce en même temps que ce qui devrait l'empêcher; autrement dit, et c'est l'un de leurs grands intérêts, plutôt que de faire réfléchir une nouvelle fois leur public au comment on se marie, elles le mettent en situation de penser le comment on vit en mariage.

Évidemment, cette typologie demeure tout à fait imparfaite et ne saurait correspondre à la grande variété des œuvres qui se sont saisies, plus ou moins longuement, de cette révolution du droit conjugal. Et si elle présente l'intérêt de rendre sensible l'importance relative que le divorce peut prendre dans les intrigues, de la simple évocation à l'enjeu principal, elle ne saurait rendre compte parfaitement d'œuvres plus complexes, qui bien souvent interrogent le principe de la théâtralité lui-même. Ainsi en va-t-il, par exemple, d'œuvres spéculaires comme Cadet Roussel misantrope et Manon repentante $e^{37}$ ou La Veille des noces ou l'Après-souper de Misantbropie et repentir ${ }^{38}$, dans lesquelles les dramaturges n'interrogent pas directement le problème du divorce mais donnent à voir aux spectateurs réels les réactions de personnages-spectateurs face à une œuvre dramatique qui elle-même traite du divorce d'autres personnages. Pour autant, il est un point où de telles œuvres, aussi originales fussent-elles, rejoignent l'ensemble du théâtre du divorce: dans leur volonté de peser sur le quotidien de leur public.

En effet, ce théâtre révolutionnaire du divorce ne se contente pas de témoigner des changements du monde extérieur en les intégrant dans ses œuvres; s'il sert de caisse de résonance à l'actualité la plus immédiate qui se déploie à l'extérieur des théâtres, il ne saurait être pour autant cantonné dans cette simple fonction d'enregistrement d'une réalité de laquelle il serait hermétiquement séparé. Le théâtre appartient pleinement à la cité et, s'il bruit du monde extérieur, il contribue lui-même, pour sa part, à alimenter ce mouvement des êtres et des choses: il est aussi un lieu dans lequel se construit le quotidien et l'avenir de la nation. De même que l'Assemblée nationale, haut lieu de la politique, fonctionne comme un théâtre, les théâtres, haut lieu de l'art dramatique, fonctionnent parfois comme une tribune politique. C'est bien évidemment le cas avec un sujet aussi intimement lié à la Révolution politique qu'est le divorce. Ainsi, loin de seulement montrer la nouvelle réalité d'un mariage dont la

sentée pour la première fois à Paris sur le Théâtre du Vaudeville le 18 mai 1793.

(35) N.J. Forgeot, Le Double Divorce, ou le Bienfait de la loi, Paris, Prault, an III (1794-1795), comédie en un acte et en vers, représentée pour la première fois à Paris sur le Théâtre de l'Égalité le 5 vendémiaire an III (26 septembre 1794).

(36) O. De Gouges, La Nécessité du divorce, ms. (1790), réédité dans Olympe de Gouges-Théâtre politique II, préfacé par G. Thiele-KNOBloch, Paris, Côté-femmes, «Des femmes dans l'histoire», 1993, pp. 143-181.
(37) J. Audé et A. Hapdé, Cadet Roussel misantrope et Manon repentante, Paris, Théâtre du Vaudeville, an VII (1799), folie en un acte et en vers, représentée à Paris sur le Théâtre des Variétés, jardin Égalité le 4 floréal an VII (23 avril 1799).

(38) H. Dorvo, La Veille des noces ou l'Aprèssouper de Misantbropie et repentir, Paris, Roussot, Cretté, an VII (1798-1797), comédie en un acte et en vers, représentée pour la première fois sur le Théâtre de Molière le 26 germinal an VII (15 avril 1799). 
pérennité n'est plus garantie par la loi, d'un mariage dont la durée de vie est soumise aux aléas d'un quotidien conjugal dont la nature se trouve profondément modifiée par le droit, ce théâtre porte un discours sur cette loi du divorce, sur sa légitimité ou, au contraire, sur ses limites et ses dangers. En ce sens, il fonctionne comme une sorte d'espace expérimental dans lequel les effets de la loi seraient observables sans danger par les spectateurs, comme une sorte de laboratoire dramatique dans lequel le divorce pourrait s'éprouver sensiblement sans conséquences réelles dans la vraie vie. Ainsi, importe-t-il de s'interroger, chaque fois qu'une œuvre mentionne cette loi nouvelle - fût-ce ponctuellement - sur le discours qu'elle porte sur sa valeur. Et de ce point de vue, force est de constater que les pièces qui en parlent le plus, qui en font un enjeu central de leur intrigue, ne sont pas toujours celles qui émettent les critiques les plus fortes ou les plus tranchées. Il suffit parfois d'une seule mention du divorce pour que ce dernier et la loi qui l'autorise soient englobés dans une critique plus large, qu'elle soit positive ou négative. Dans Les Parvenus d'aujourd'bui ou le Véritable $A m i^{39}$ de Trial-Latour, la simple mention du divorce de Farollet, un ancien domestique devenu accapareur après avoir dénoncé et volé son maître, aboutit à associer dans la condamnation la loi qui permet un agiotage sur le mariage et l'immoralité qui nourrit la spéculation sur les prix et l'enrichissement de profiteurs peu scrupuleux - le véritable sujet de l'œuvre.

Ainsi, les représentations dramatiques apparaissent-elles parfois comme de véritables armes de lutte contre une loi jugée dangereuse pour les individus comme pour la collectivité ou, inversement, contre ceux-là mêmes qui refusent la dissolubilité légale du mariage; elles sont alors, pour reprendre une terminologie de l'époque, antidivorciaires ou divorciaires. Amar du Rivier, par exemple, dans son drame intitulé Les Suites et les dangers du divorce ${ }^{40}$, nous montre les conséquences tragiques que peut entraîner cette loi inique: son hérö̈ne, après avoir injustement divorcé de son époux vertueux que des rumeurs accusaient d'adultère, s'est remariée à un homme violent qu'à présent elle fuit avec sa fille; et la pièce de s'achever, après un fâcheux concours de circonstances, par la double disparition de l'ancien et du nouveau mari qui se sont entre-tués en duel. La leçon, rappelée par Mme d'Étanges au dénouement de la pièce, ne saurait être plus claire: «les nœuds les plus chers, les plus sacrés de tous, / $\mathrm{Ne}$ se brisent jamais sans remords et sans crime» ${ }^{41}$. Inversement, d'autres œuvres soulignent le grand intérêt de cette loi qui permet de briser des mariages conclus sans l'assentiment des deux époux, des mariages sans amour qui semblent condamner les conjoints à l'adultère ou à la frustration amoureuse. Il s'agit explicitement du propos de Forgeot lorsqu'il écrit sa comédie Le Double Divorce ou le Bienfait de la loi $i^{42}$, tout, dans cette pièce, étant fait pour souligner le bénéfice apporté par cette loi, qui permet ici, sous l'influence bienfaitrice de Lucinde, de libérer Dorlis et Cécile de deux unions de raison afin de les promettre l'un à l'autre.

Pourtant, force est de constater que le théâtre du divorce ne cherche pas toujours, loin s'en faut, à agir sur le droit conjugal en démontrant par la fiction son intérêt ou ses dangers, autrement dit en convainquant les spectateurs de la nécessité éventuelle de peser à leur tour sur les législateurs pour qu'ils le modifient. En réalité, la plupart des œuvres dramatiques abordant la question du divorce s'immiscent plus intimement dans la vie de leurs spectateurs en leur apprenant comment comprendre cette loi et surtout comment l'utiliser. C'est tout le sens des nombreuses pièces sur le

(39) Trial-Latour, Les Parvenus d'aujourd'bui ou le Véritable Ami, Le Havre, Faure, 1797, comédie en trois actes et en prose, représentée pour la première fois sur le théâtre du Havre en 1796.
(40) J.-A. Amar DU Rivier, op. cit.

(41) Ibid., p. 49.

(42) N.J. Forgeot, Le Double Divorce, ou le Bienfait de la loi, cit. 
divorce sans divorce écrites pendant la période révolutionnaire, de ces œuvres dans lesquelles la réconciliation finale des époux les conduit finalement à abandonner leur projet de séparation voire à revenir sur un divorce déjà prononcé. En effet, à bien étudier ce corpus, il apparait que les dramaturges cherchent plus souvent à seconder - plutôt qu'à influencer - la volonté des législateurs qui, rappelons-le, n’ont accordé aux Français une loi aussi libérale qu'avec l'intention clairement exprimée de régénérer la famille et par-là de rendre les mariages plus heureux, autrement dit de rendre les divorces les plus rares possibles. En mettant en scène des couples qui se réunissent et renoncent à une rupture un temps envisagée, ces œuvres définissent, au fil des situations et des dialogues qu'elles imaginent, une morale du divorce bien faite pour en limiter les abus; elles mettent distinctement en place une pédagogie dramatique supposée faire comprendre aux spectateurs l'esprit plutôt que la lettre de la loi de 1792: ce qu'ils doivent faire plutôt que ce qu'ils peuvent faire. En cela, le théâtre, devenu école du spectateur, remplit tout à fait le rôle de précepteur que les révolutionnaires ont voulu lui assigner; il vient même en soutien d'une loi dont l'idéalisme transparait dans l'écart entre la vertu de ses ambitions - moraliser le mariage - et les moyens qu'elle se donne pour y parvenir - libéraliser sans contraintes la pratique du divorce.

La mise en évidence de cette morale portée par le théâtre du divorce montre bien comment il tente d'une certaine manière de résorber ce flottement entre principes et réalité, dans lequel l'efficacité de la loi risque de se diluer. En retrouvant au plus près l'esprit qui animait les législateurs, qui n'étaient pas aussi révolutionnaires que la loi votée pourrait le laisser penser, ces pièces viennent en quelque sorte faire le lien entre un droit libéral au divorce qu'elles défendent dans son principe et son usage moralisé qu'elles enseignent au gré de leurs intrigues. Cette morale, qui s'incarne de manière toujours originale sous la plume de chaque dramaturge, peut néanmoins être ramenée à trois grands principes qui, s'ils ne sont pas toujours présents dans les pièces, reviennent néanmoins suffisamment souvent pour prétendre caractériser ce corpus. Le premier, dont la pièce Le Mur mitoyen ou le Divorce manquét3 est un bon exemple, nous enseigne que si le mariage ne doit plus être décidé par les parents sans l'avis des enfants, il n'en reste pas moins que ces derniers ont tout intérêt à obéir aux premiers, d'autant plus volontiers que l'amour pourra toujours suivre le mariage et donner raison aux parents. Ainsi Linval, marié par sa famille, découvre-t-il cette vérité en renonçant à divorcer de sa femme, dont il tombe amoureux en la prenant pour une autre. Le second principe moral défendu par ce théâtre du divorce concerne les rapports entre les hommes et les femmes dans le couple. Si la loi du divorce installe une démocratie sexuelle de fait au sein du mariage, ces œuvres prennent néanmoins soin de rappeler qu'un couple équilibré et harmonieux se fonde plus volontiers sur l'obéissance de l'épouse invitée à toujours (re)gagner l'attention et la fidélité de son mari par le charme, et sur la bienveillance de l'époux qui ne doit jamais abuser de ses pouvoirs - qui paraissent lui rester malgré la loi. C'est la leçon donnée à sa fille et aux spectateurs par l'épouse de la comédie Les Moeurs ou le Divorce ${ }^{44}$ de PigaultLebrun: parvenant à reconquérir le cœur de son époux volage et donc à échapper au divorce à force de séduction et de soumission, elle indique le chemin à suivre. Enfin, le troisième pilier de cette morale conjugale concerne la nature même de ce qui fonde le mariage. Nous avons souligné combien le droit révolutionnaire du divorce rend

(43) P.-Y. Barké et N. BourgueIL, op. cit.

(44) Ch.A.G. Pigault-Lebrun, Les Mours ou le Divorce, Paris, chez Barba, an III (1794-1795), comédie en un acte et en prose, représentée pour la première fois à Paris sur le Théâtre de la Cité la quatrième sans-culottide an II (20 septembre 1794). 
possible le mariage d'amour. Or une telle union risque de ne durer que le temps du sentiment et ainsi, l'amour étant par essence versatile, de souffrir d'une instabilité chronique préjudiciable aux ambitions du législateur. Les auteurs qui nous intéressent ici ont parfaitement perçu un tel danger et ont donc pris soin de montrer dans leurs productions combien un mariage solide et heureux s'appuie plus volontiers sur une tendresse conjugale - qui peut être le résultat d'un amour assagi - et sur l'amour parental. Il ne saurait être question pour eux d'abandonner le mariage aux aléas de la passion imprévisible et incontrôlable. C'est ce dont Licas prend conscience dans La Double Réconciliation ${ }^{45}$ de Dupont de Lille, quand, après avoir voulu divorcer par amour pour une autre, il décide finalement de retrouver son épouse enceinte.

Malgré sa défense du principe de la loi du divorce, ce théâtre professe donc une morale conjugale peu suspecte de vouloir détruire la cellule familiale; ce faisant, il retrouve les motivations des législateurs et il s'associe même à eux pour changer, avec ses armes, le quotidien des spectateurs.

Au terme de ce parcours, il conviendrait de nuancer la portée de ce propos afin de ne pas induire une perception faussée de l'époque et de la place qu'y occupe le théâtre, et ainsi de ne pas en affaiblir la teneur. S'il est clair que le droit du divorce est devenu dès 1789 une nouvelle thématique dramatique - ce qu'il ne cessera plus d'être -, et que les dramaturges s'en sont saisis en s'intéressant à ses usages - qu'ils ont voulu moraliser - plutôt qu'à sa valeur intrinsèque, il ne faut pas pour autant y voir un fait culturel massif dont les conséquences auraient irrigué le quotidien de tous les Français. En effet, à l'instar de la loi du divorce, les représentations de ce théâtre n'ont concerné qu'une petite partie, essentiellement parisienne, de la population française. Aussi importe-t-il de se montrer prudent lorsque l'on affirme que ces pièces faisaient résonner sur la scène un quotidien profondément transformé par la loi du divorce et qu'en retour elles cherchaient à peser sur ce quotidien par ses préceptes moraux: nombreux sont ceux sans doute qui avaient à peine connaissance de cette loi, que ce fût directement ou par la voix du théâtre.

Pour autant, face à cet événement juridique majeur, l'importance du fait culturel demeure et nous en apprend un peu plus sur la manière dont le théâtre révolutionnaire pensait lui-même sa place dans la société.

PHILIPPE CORNO

(45) B. Dupont de Lille, La Double Réconciliation, Paris, chez Michel, An VII (1798-1799), opéra-vaudeville représenté pour la première fois, à Paris, sur le Théâtre des Jeunes Artistes le 5 thermidor an IV (23 juillet 1796). 